\title{
Ophthalmic rosacea in a patient with Takayasu disease
}

\section{Sameh Sayhi', Sameh Mezri², Nadia Ben Abdelhafidh'}

${ }^{1}$ Internal Medicine Department, Military Hospital of Tunis, Tunis, Tunisia, ${ }^{2}$ ENT Surgery Department, Military Hospital of Tunis, Tunis, Tunisia

Corresponding author: Dr. Sameh Sayhi, E-mail: sameh_sayhi@yahoo.fr

Rosacea is a chronic dermatological disease that may affect the ocular structures up to 6-72\% [1] of all cases with important visual consequences for affected patients. Therefore, an early diagnosis associated with adequate treatment is important [2].

The aim of this study is to report an original observation of ophthalmic rosacea in a patient with Takayasu disease.

We report the case of 49-year-old women with Takayasu disease treated by methotrexate $20 \mathrm{mg}$ /day who consulted for eye redness, tearing, and decreased visual acuity. The ophthalmological examination revealed a visual acuity (VA) of 9/10, keratoconjunctivitis + blepharitis (Fig. 1). He was diagnosed with ocular rosacea and treated with doxycycline and topical tacrolimus cream.

Ocular rosacea includes multiple ophthalmic manifestations ranging from inflammation of the eyelid margin and blepharitis to serious corneal affectations. An early diagnosis associated with adequate treatment visual allows avoiding sequelae.

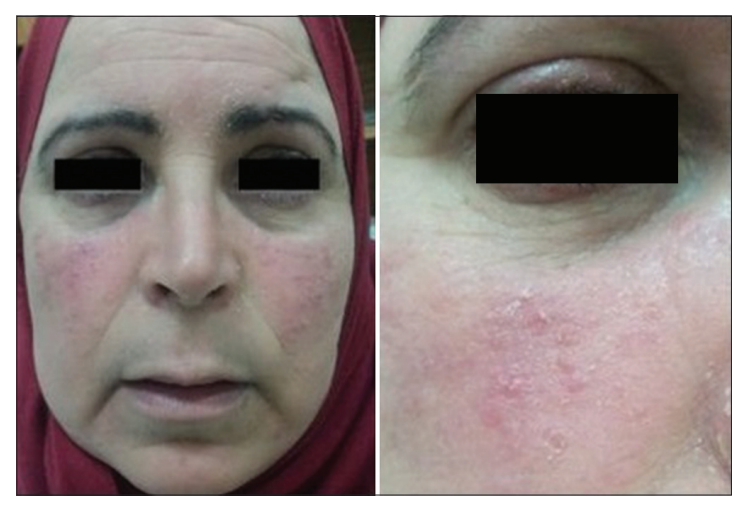

Figure 1: Blephartis and conjonctivitis in patient with rosacea.

\section{REFERENCES}

1. Vieira AC, Höfling-Lima AL, Mannis MJ. Ocular rosacea-a review. Arq Bras Oftalmol. 2012;75:363-9.

2. Gloria López-Valverde, Elena Garcia-Martin, Jos, Manuel Larrosa Povés, Vicente Polo-Llorens, Luis E. Pablo-Júlvez. Therapeutical Management for ocular Rosacea. Case Rep Ophthalmol. 2016;7:237-42.

Copyright by Sameh Sayhi, et al. This is an open-access article distributed under the terms of the Creative Commons Attribution License, which permits unrestricted use, distribution, and reproduction in any medium, provided the original author and source are credited.

Source of Support: Nil, Conflict of Interest: None declared. 